\title{
Tinnitus revival during COVID-19 lockdown: how to deal with it?
}

\author{
Roberta Anzivino $^{1}\left(\mathbb{D} \cdot\right.$ Pasqua Irene Sciancalepore $^{2} \cdot$ Paolo Petrone $^{1} \cdot$ Alessandra D $^{\prime}$ Elia $^{1} \cdot$ Domenico Petrone $^{1}$. \\ Nicola Quaranta ${ }^{2}$
}

Received: 30 May 2020 / Accepted: 16 June 2020 / Published online: 22 June 2020

(c) Springer-Verlag GmbH Germany, part of Springer Nature 2020

Keywords Tinnitus $\cdot$ COVID-19 $\cdot$ Smartphone applications $\cdot$ Cognitive behavioural therapy $\cdot$ Retraining therapy $\cdot$ Sound therapy

To the Editor,

The novel Coronavirus Disease, officially designated as COVID-19 by the WHO, is a serious issue for public health. To contain the COVID-19, the Italian Government stated on March 9th 2020 the prohibition of any movement throughout the national area unless for work/health reasons and the obligation to remain as much as possible inside one's own home. With the start of the so-called "Phase Two" on May 4 th 2020, circulation within the same region was allowed again, due to the progressive slowdown of the outbreak.

Therefore, since lockdown measures were relaxed and access to the emergency room or ENT clinic became less worrying for patients, specialists of Otolaryngology Units in Bari (Italy) observed an increase in the amount of subjects complaining of the revival of intense tinnitus. We attempt in this letter to focus on patients affected by chronic subjective tinnitus, that already had a diagnosis and self-stabilized without a massive treatment.

Research studies have reported tinnitus wide impact on quality of life of subjects experiencing it, involving their emotional state, concentration and sleep quality; at this regard, Tinnitus Handicap Inventory (THI) is a self-reported tool regularly used to quantify the grade of perceived handicap as slight (0-16), mild (18-36), moderate (38-56), severe

This comment refers to the article available online at https://doi. org/10.1007/s00405-019-05743-8.

Roberta Anzivino

roberta.anzivino@gmail.com

1 Otolaryngology Unit, Di Venere Hospital, Via Ospedale Di Venere 1, 70131 Bari, BA, Italy

2 Otolaryngology Unit, Department of BMS, Neuroscience and Sensory Organs, University of Bari “Aldo Moro”, Bari, Italy
(58-76) or catastrophic (78-100) on the basis of 25 questions [1].

During the past 2 weeks, we have collected data from 16 patients among our population of chronic sufferers: THI observed was moderate in $62.5 \%$ and severe in $18.75 \%$ of cases, catastrophic in $12.5 \%$ and mild in $6.25 \%$ of subjects. Interestingly, the grade of handicap resulted increased by one-level in 12 out of 16 patients (75\%); in particular, THI shifted from mild to moderate in 9 patients and from moderate to severe in 3 patients.

As shown in the literature, tinnitus generation, maintenance and recrudescence are still debated. A cortical reorganization secondary to sensory deprivation has been proposed as one on the most frequent cause of tinnitus [2]. The avoidance of silence and acoustic masking have been proposed as effective measures to overcome sensory deprivation and increase masking of the symptom [3]. It is reasonable to think that, during the lockdown, the absence of environmental masking sounds from everyday life may have enhanced the tinnitus perception. Furthermore, proneness to worry and incoming stress during pandemic could be included as further potential risk factors for tinnitus worsening.

As proficiently reviewed in a recent work [4], some internet/smartphone-based applications provide in tinnitus patients adequate counseling and interactive information together with sound therapy. As brain networks implicated in adaptive responses to sound stimuli and to worry are shared in many cases, an early decrease of anxiety status may release neural resources crucial for tinnitus habituation/ distress perception [5]. In general, interactive platforms have been widely implemented during lockdown period due to the forced lack of real personal and working relationships; since smart-working seems to be successful for future plans, the development of smart applications and mobile services 
in the health care field may be promising in terms of costeffectiveness, tolerability and simplicity of use.

Funding None.

\section{Compliance with ethical standards}

Conflict of interest The authors report no conflict of interest and compliance with ethical standards. They did not receive any funding nor economical support for this study.

\section{References}

1. Monzani D, Genovese E, Marrara A et al (2008) Validity of the Italian adaptation of the Tinnitus Handicap Inventory; focus on quality of life and psychological distress in tinnitus-sufferers. Acta Otorhinolaryngol Ital 28(3):126-134
2. Noreña AJ, Farley BJ (2013) Tinnitus-related neural activity: theories of generation, propagation, and centralization. Hear Res 295:161-171. https://doi.org/10.1016/j.heares.2012.09.010

3. Zenner HP, Delb W, Kröner-Herwig B et al (2017) A multidisciplinary systematic review of the treatment for chronic idiopathic tinnitus. Eur ArchOtorhinolaryngol 274(5):2079-2091. https:// doi.org/10.1007/s00405-016-4401-y

4. Nagaraj MK, Prabhu P (2020) Internet/smartphone-based applications for the treatment of tinnitus: a systematic review. Eur Arch Otorhinolaryngol 277(3):649-657. https://doi.org/10.1007/s0040 5-019-05743-8

5. Kraus KS, Canlon B (2012) Neuronal connectivity and interactions between the auditory and limbic systems. Effects of noise and tinnitus. Hear Res 288(1-2):34-46. https://doi.org/10.1016/j. heares.2012.02.009

Publisher's Note Springer Nature remains neutral with regard to jurisdictional claims in published maps and institutional affiliations. 